This is the submitted version of the article:

Li F., Mawby P., Song Q., Perez-Tomas A., Shah V., Sharma Y., Hamilton D., Fisher C., Gammon P., Jennings M.. A First Evaluation of Thick Oxide 3C-SiC MOS Capacitors Reliability. IEEE Transactions on Electron Devices, (2020). 67. 8935512: 237 - . 10.1109/TED. 2019.2954911.

Available at: https://dx.doi.org/10.1109/TED.2019.2954911 


\title{
A first evaluation of thick oxide 3C-SiC MOS capacitors reliability
}

\author{
Fan Li, Qiu Song, Amador Perez-Tomas, Vishal Shah, Yogesh Sharma, \\ Dean Hamilton, Craig Fisher, Peter Gammon, Mike Jennings, and Phil Mawby
}

\begin{abstract}
Despite the recent advances in 3C-SiC technology, there is a lack of statistical analysis on the reliability of $\mathrm{SiO}_{2}$ layers on $3 \mathrm{C}-\mathrm{SiC}$, which is crucial in power MOS device developments. This paper presents a comprehensive study of the medium and long-term time-dependent dielectric breakdown (TDDB) of $65 \mathrm{~nm}$ thick $\mathrm{SiO}_{2}$ layers thermally grown on a state-of-the-art $3 \mathrm{C}-\mathrm{SiC} / \mathrm{Si}$ wafer. Fowler-Nordheim (F-N) tunnelling is observed above 7 $\mathrm{MV} / \mathrm{cm}$ and an effective barrier height of $3.7 \mathrm{eV}$ is obtained, which is highest known for native $\mathrm{SiO}_{2}$ layers grown on the semiconductor substrate. The observed dependence of the oxide reliability on the gate active area suggests the oxide quality has not reached the intrinsic level. Three failure mechanisms were identified, confirmed by both medium and long-term results. Whereas two of them are likely due to extrinsic defects from material quality and fabrication steps, the one dominating the high field $(>8.5 \mathrm{MV} / \mathrm{cm})$ should be attributed to the electron impact ionization within $\mathrm{SiO}_{2}$. At room temperature, the field acceleration factor is found to be $\approx 0.906 \mathrm{dec} /(\mathrm{MV} / \mathrm{cm})$ for high fields, and the projected life-time exceeds 10 years at $4.5 \mathrm{MV} / \mathrm{cm}$.
\end{abstract}

Index Terms - Reliability, 3C-SiC, MOS capacitor, TDDB, failure mechanism.

\section{INTRODUCTION}

$\mathrm{S}_{\mathrm{p}}$ LICON CARBIDE (SiC) can provide better electrical performance than $\mathrm{Si}$ in more harsh operation conditions (high power, high frequency and high temperature), thus have been considered as the next generation substrates for power devices. 4H-SiC Schottky barrier diodes (SBD), JFETs, and MOSFETs are commercially available now. The 4H-SiC SBDs proved to be a success in boost converters, whereas the switches (JFETs or MOSFETs) have not been widely accepted as their Si counterparts.

Compared with other wide band gap (WBG) semiconductors, $\mathrm{SiC}$ has the advantage that $\mathrm{SiO}_{2}$ layers can be directly thermally grown on the epilayer as the gate dielectric for MOS devices. The reliability of this $\mathrm{SiO}_{2}$ layer is a major attribute of a MOSFET development and the required lifetime of power

This paragraph of the first footnote will contain the date on which you submitted your paper for review. This work was part of the Challenge project, founded by European Commission, ID 720827.

F. Li, Q. Song, C.A. Fisher, V.A. Shah, P.M. Gammon, and P.A. Mawby are with the School of Engineering, University of Warwick, Coventry, CV4 7AL, UK. (e-mail: f.li.3@warwick.ac.uk).

A. Perez-Tomas is with Catalan Institute of Nanoscience and Nanotechnology (ICN2), CSIC and The Barcelona Institute of Science and Technology, Campus UAB, Bellaterra, 08193 Barcelona, Spain. electronics systems can reach up to 30 years [1], thus gate dielectrics are expected to have a similar lifetime. In the early days of the $\mathrm{Si}$ industry, the $\mathrm{SiO}_{2}$ on $\mathrm{Si}$ system was intensively studied and is now well developed compared with investigations performed on $4 \mathrm{H}-\mathrm{SiC}$ [2-12]. Even now, the gate oxide reliability of large active $4 \mathrm{H}-\mathrm{SiC}$ MOS devices (5-50 $\mathrm{mm}^{2}$ ) is still not as good as the early Si devices with comparable gate oxide area [13]. Regardless, in the last decade, a great improvement of the lifetime of $\mathrm{SiO}_{2}$ on $4 \mathrm{H}-\mathrm{SiC}$ has been seen, from below $1000 \mathrm{~s}\left(6 \mathrm{MV} / \mathrm{cm}, 350{ }^{\circ} \mathrm{C}\right)$ [3] to most recently $2300 \mathrm{~h}\left(6 \mathrm{MV} / \mathrm{cm}, 250^{\circ} \mathrm{C}\right)[6]$. Yet it was believed impossible to reach the stability of the $\mathrm{Si} / \mathrm{SiO}_{2}$ interface, due to an inherent smaller conduction bands offset $\Delta E_{C}$ between $4 \mathrm{H}-\mathrm{SiC}$ and $\mathrm{SiO}_{2}$ [4]. In the high field condition, the oxide intrinsic degradation is believed to be dominated by F-N tunnelling [14], and the affinity difference at the semiconductor/oxide interface is the potential barrier $\Phi_{B}$ for such tunnelling. In a strong inversion case such as the MOSFET being switched on, $\Phi_{B} \approx \Delta E_{C}$ [4]. The theoretical value of $\Delta E_{C}$ for $4 \mathrm{H}-\mathrm{SiC} / \mathrm{SiO}_{2}$ is $2.7 \mathrm{eV}$ and had recently been experimentally reported [15], whereas the value for $\mathrm{Si}$ is $3.2 \mathrm{eV}$, and for $3 \mathrm{C}-\mathrm{SiC} 3.7 \mathrm{eV}$ [16], potentially the most stable among the three. Moreover, the smaller bandgap means most of the traps troubling the $4 \mathrm{H}-\mathrm{SiC} / \mathrm{SiO}_{2}$ interface are located in the conduction band of $3 \mathrm{C}-\mathrm{SiC}$, namely not contributing to degrade the channel mobility, thus lower channel resistance for a MOSFET. 3C-SiC technology is not yet as popular as $4 \mathrm{H}-\mathrm{SiC}$, mainly due to the lack of device grade, high quality $3 \mathrm{C}-\mathrm{SiC}$ substrates. However, the ability to deposit $3 \mathrm{C}$-SiC on large area $\mathrm{Si}$ wafers (6-inch $3 \mathrm{C}$-SiC on $\mathrm{Si}$ wafers demonstrated 20 years ago [17]) makes it attractive in terms of cost-effective. In the last ten years, there have been many improvements in 3C-SiC substrate preparation [18-24] that a defect density below $400 \mathrm{~cm}^{-1}$ became possible [24]. There were also further developments in $3 \mathrm{C}$-SiC device processing, including implantation [25, 26], oxidation [27-29], and metallisation [30, 31]. $600 \mathrm{~V}$ vertical power MOSFET was demonstrated with a specific on-resistance of $8.2 \mathrm{~m} \Omega . \mathrm{cm}^{2}$ [32].

Y. K. Sharma is with Dynex Semiconductor Ltd, Doddington Rd, Lincoln LN6 3LF, UK

D.P. Hamilton is with Institute of Engineering Sciences, De Montfort University, Leicester, LE1 9BH, UK.

M.R. Jennings is with College of Engineering, Swansea University, Swansea, SA1 8EN, UK. 
Regarding the $3 \mathrm{C}-\mathrm{SiC} / \mathrm{SiO}_{2}$ interface, some study was performed on the interface trap density [33, 34] and channel mobility $[29,35,36]$, whereas very little on the reliability. In [16], dielectric breakdown of very thin $(6-7 \mathrm{~nm})$ and small area (100 nm diameter) thermally grown $\mathrm{SiO}_{2}$ on $3 \mathrm{C}-\mathrm{SiC}(111)$ was studied by Time Dependent Dielectric Breakdown (TDDB) test at $10 \mathrm{~V}(\approx 15 \mathrm{MV} / \mathrm{cm})$, and Weibull slope $\beta$ values of 4.4-5.1 were obtained. This is close to the performance of $\mathrm{SiO}_{2}$ layers on $\mathrm{Si}$ with similar thickness. However, for thin oxides the leakage current stays almost constant until failure, which is in contrast to thick oxides, where electron/hole trapping cause the current level to change a lot during stress [14]. Since the tunnelling current generates defects in the oxide, leading to their eventual degradation [4], thin and thick $\mathrm{SiO}_{2}$ layers tend to have different failure mechanisms. Also, under the same electric field, carriers in thicker oxides are more likely to gain a higher energy for impact ionization [9], which is often considered as another potential cause of oxide failure. Practical power devices usually have thick $(>50 \mathrm{~nm}) \mathrm{SiO}_{2}$ layer in order to sustain the gate voltage $[4,7]$. To the best of our knowledge, the reliability of thick $\mathrm{SiO}_{2}$ on $3 \mathrm{C}-\mathrm{SiC}$ has not been reported.

In this paper, $3 \mathrm{C}$-SiC MOS capacitors were fabricated with $\approx 65 \mathrm{~nm}$ thermally grown $\mathrm{SiO}_{2}$ layers as the gate dielectric. I-V characterisation was used to study the effective barrier height at the $3 \mathrm{C}-\mathrm{SiC} / \mathrm{SiO}_{2}$ interface. Medium-term test lasting weeks was then performed and the failure distribution was obtained for a wide electric field range of $4.5-10.5 \mathrm{MV} / \mathrm{cm}$. Following the medium-term results, long-term TDDB tests lasting months were conducted for $6,7.5,8.5$ and $9 \mathrm{MV} / \mathrm{cm}$. All characterisations were performed at room temperature. Based on the failure distribution and TDDB plots, Weibull models were used to predict the fabricated MOS capacitors failure rate and lifetime.

\section{EXPERIMENTAL}

$10 \mu \mathrm{m}$ thick unintentionally doped $\left(<1 \times 10^{16} \mathrm{~cm}^{-3}\right)$ N-type $3 \mathrm{C}$-SiC(100) layers were epitaxy grown on-axis from a 4-inch $\mathrm{Si}(100)$ substrate. The as-grown $3 \mathrm{C}-\mathrm{SiC}$ epilayer was treated with a chemical mechanical polishing (CMP) step in order to obtain a low surface root mean square roughness $\approx 0.2 \mathrm{~nm}$, measured by atomic force microscopy (AFM). Since it is the $3 \mathrm{C}-\mathrm{SiC} / \mathrm{SiO}_{2}$ interface that is of interests here, lateral MOS capacitors were fabricated in order to minimize the disturbing electrical effects from the $3 \mathrm{C}-\mathrm{SiC} / \mathrm{Si}$ heterojunction. For ohmic contact, nitrogen was selectively implanted to form a $300 \mathrm{~nm}$ deep box profile with the peak concentration of $\approx 5 \times 10^{20} \mathrm{~cm}^{-3}$, and a post implantation anneal was performed at $1350{ }^{\circ} \mathrm{C}$ for 2 hours in Ar atmosphere. No surface capping layer was used during the post implantation anneal. Our previously study [26] shows that, this will lead to a $\approx 15 \%$ activation of the implanted nitrogen dopants. After the post implantation anneal, solvent cleans were performed (acetone, propanol, acetone, and methanol, each step 5 mins) in an ultrasonic bath. Following that, a two-stage acid clean was applied, first piranha $\left(\mathrm{H}_{2} \mathrm{SO}_{4}: \mathrm{H}_{2} \mathrm{O}_{2}=3: 1,15\right.$ mins) then RCA (RCA1 15 mins+ RCA2 15 mins). A $2 \mu \mathrm{m}$ thick $\mathrm{SiO}_{2}$ layer was deposited via low pressure chemical vapour deposition as the surface passivation dielectric. Photo lithography and reactive ion etching (RIE) was used to selectively etch the deposited $2 \mu \mathrm{m} \mathrm{SiO}_{2}$ down to the $3 \mathrm{C}-\mathrm{SiC}$ surface, where the gate thermal oxidation occurred. Dry oxidation $\left(1200{ }^{\circ} \mathrm{C}, 10 \mathrm{mins}, 1 \mathrm{slm} \mathrm{O}_{2}\right)$ followed by a post oxidation anneal in $\mathrm{N}_{2} \mathrm{O}\left(1200{ }^{\circ} \mathrm{C}, 120\right.$ mins, $\left.1 \mathrm{slm} \mathrm{N}_{2} \mathrm{O}\right)$ was performed to obtain the gate oxide. The final gate oxide was around $65 \mathrm{~nm}$ thick, measured by the ellipsometry. In our previous investigations, no significant degradation in the $3 \mathrm{C}$ $\mathrm{SiC}$ surface roughness was observed following AFM evaluations after post implantation anneal [26] and thermal oxidation [27]. Ohmic metallisation was obtained by RIE etching the passivation oxide and evaporating first a $30 \mathrm{~nm} \mathrm{Ti}$ layer, followed by a $100 \mathrm{~nm} \mathrm{Ni}$ layer on the $\mathrm{N}+$ regions. A rapid thermal anneal at $1000{ }^{\circ} \mathrm{C}$ for 2 mins in $\mathrm{Ar}$ was performed to form the ohmic contact with specific contact resistance below $1 \times 10^{-5} \Omega . \mathrm{cm}^{2}$ [31]. Finally, the gate contact was defined by selectively evaporating $500 \mathrm{~nm}$ thick $\mathrm{Al}$ above the gate oxide region. The final device has a circular gate active area, and a cross section view of the structure is shown in Fig. 1. MOS capacitors with three gate diameters were fabricated, namely $\Phi 100 \mu \mathrm{m}, \Phi 200 \mu \mathrm{m}$ and $\Phi 400 \mu \mathrm{m}$.

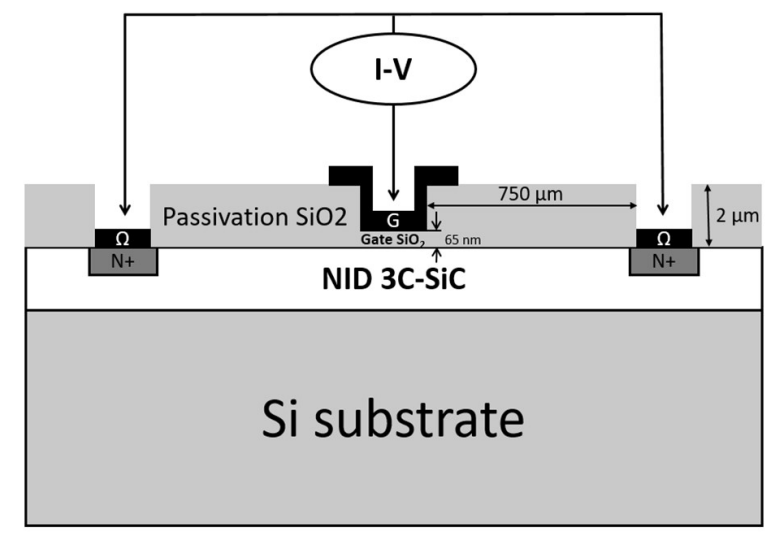

Fig. 1. A schematic cross section view of the fabricated circular lateral 3CSiC MOS capacitors, dimensions are not to scale.

\section{RESULTS AND DISCUSSIONS}

\section{A. Capacitance-voltage (C-V) characterisation}

Room temperature C-V (1 kHz and $1 \mathrm{MHz}$ ) characterisations were performed on these MOS capacitors using a LCR meter. A negative flat band voltage was observed as shown in Fig. 2, which is also commonly reported in the literature [32, 36, 37]. It is generally believed to be caused by the nature of donor-like states at the $3 \mathrm{C}-\mathrm{SiC} / \mathrm{SiO}_{2}$ interface, which have a lower density than $4 \mathrm{H}-\mathrm{SiC}$ but are positively charged [38]. The effective fixed charge density was calculated to be around $2.1 \times 10^{12} \mathrm{~cm}^{-2}$ according to the flat band shift from the ideal value. This value is typical among lately reported results $[16,28]$. Using the highlow method [39], the interface trap density at $0.2 \mathrm{eV}$ from the $3 \mathrm{C}$-SiC conduction band edge was extracted to be $2.5 \times 10^{11} \mathrm{~cm}^{-}$ ${ }^{2} \mathrm{eV}^{-1}$, which is among the lowest values reported in recent literatures $[28,34,40]$. 


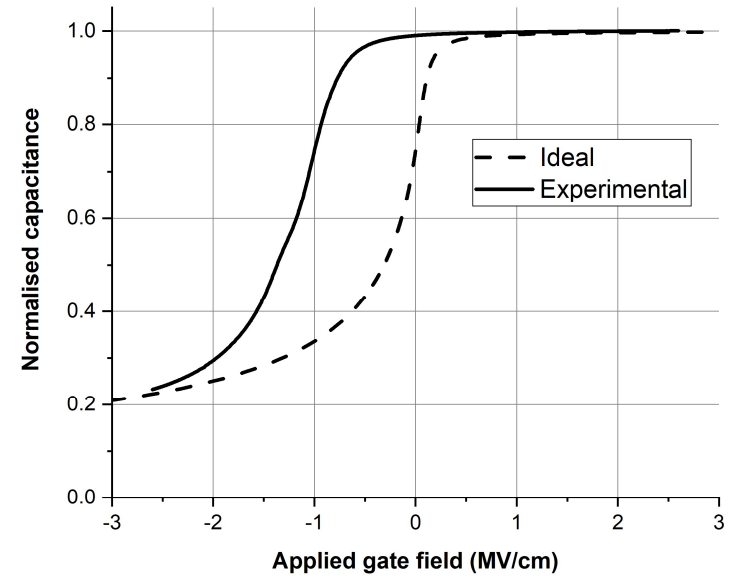

Fig. 2. Experimental $(1 \mathrm{MHz})$ and ideal $\mathrm{C}-\mathrm{V}$ characterisations of the fabricated lateral MOS-C.

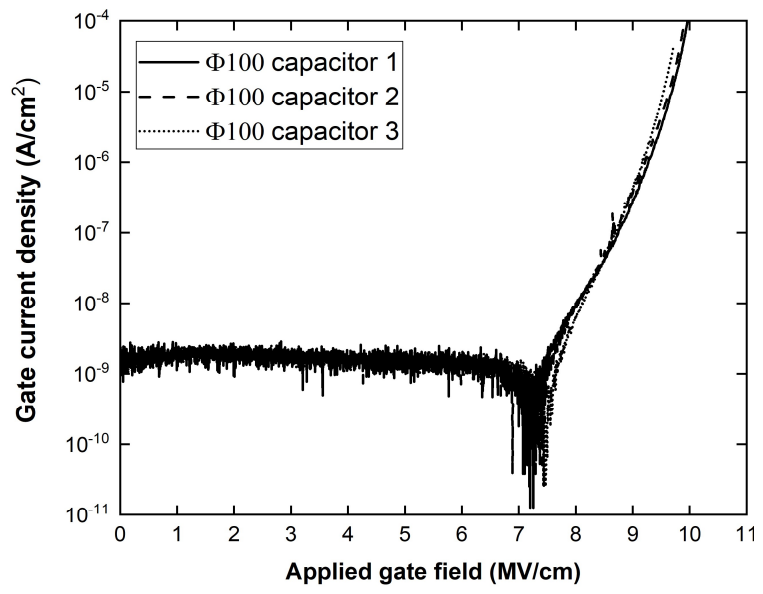

Fig. 3. Representative gate leakage current densities as a function of the electric field in the oxide for the $\Phi 100$ MOS capacitors.

\section{B. Dielectric breakdown and $F-N$ tunnelling}

By performing room temperature $\mathrm{I}-\mathrm{V}$ measurements using a Keysight B1500 parameter analyser, the leakage current density of $\Phi 100$ devices as a function of the electric field in the oxide is obtained and shown in Fig. 3. As can be seen in Fig. 3, when the electric field reaches $\approx 7 \mathrm{MV} / \mathrm{cm}$, the gate leakage current density becomes dominated by F-N tunnelling, which usually comes into effect at a lower electric field of 5-5.5 MV/cm for 4H-SiC MOS gate oxides [7-12, 15]. The effective barrier height $\Phi_{B}$ can be obtained by fitting the linear region of the experimental " $\ln \left(J / E_{o x}{ }^{2}\right)$ vs $1 / E_{o x}$ " plot to the following expression [15]:

$$
\ln \left(\frac{J}{E_{O x^{2}}{ }^{2}}\right)=\frac{q^{3}\left(\frac{m_{0}}{m_{O x}}\right)}{8 \pi h \Phi_{B}}-\frac{8 \pi \sqrt{2 m_{O x} \Phi_{B}{ }^{3}}}{3 q h E_{O x}}
$$

where $J$ is the gate leakage current density, $E_{o x}$ is the electric field within the gate oxide, $m_{0}$ is the free electron mass, $m_{o x}=0.42 m_{0}$ [41] is the effective electron mass, $q$ is the unit electron charge, and $h$ is the Plank constant. The experimental effective barrier height $\Phi_{B}$ was obtained as $3.65-3.71 \mathrm{eV}$ for the representative results shown in Fig. 3, approaching the theoretical value of $3.7 \mathrm{eV}$.

\section{Medium-term reliability and failure distribution}

We investigated the failure distribution of these MOS capacitors in a wide field range of 4.5-10.5 MV/cm. For each studied electric field, the gate bias $V_{G}$ was kept constant for 1 hour before increasing to the next value. More details about this method can be found in [1].

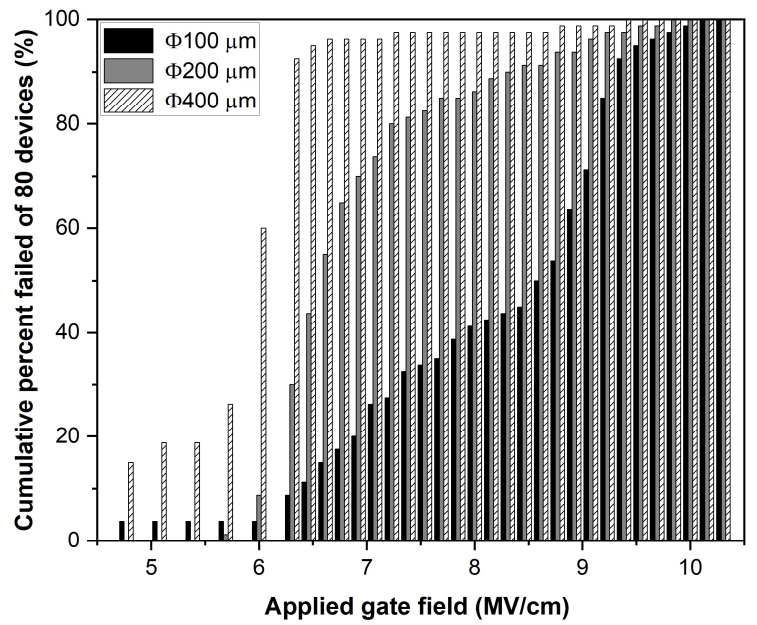

Fig. 4. The failure distribution of $\Phi 100, \Phi 200$ and $\Phi 400$ MOS capacitors in the electric field range of 4.5-10.5 MV/cm.

For each gate diameter, 80 MOS capacitors were tested. As can be seen in Fig. 4, the failure distribution varies considerably with the gate diameter that, larger devices tend to fail more easily than smaller ones as the gate bias elevates. Similar area dependence was observed also in 4H-SiC TDDB results (area of $100 \times 100-200 \times 800 \mu^{2}$ ) [7]. At least three mechanisms are recognisable from the failure distribution of the $\Phi 100 \mathrm{MOS}$ capacitors. Neglecting the 3 premature failures at $4.5 \mathrm{MV} / \mathrm{cm}$, it can be seen that between $6 \mathrm{MV} / \mathrm{cm}$ and $7.5 \mathrm{MV} / \mathrm{cm}$, the number of failures accumulate gradually. In this low field range, failure is crucially determined by the number of extrinsic defects per device (namely gate active area) and defined as mechanism I. From the literature, these first priority defects may be pre-existing basal plane dislocations in the $3 \mathrm{C}-\mathrm{SiC}$ epilayer [42], or local step-bunching edges where dielectric break down can be accelerated [16]. Reaching $7.5 \mathrm{MV} / \mathrm{cm}$, it seems that almost all mechanism I failures have occurred, and the increase of total failure number slows down, suggesting a new mechanism II becoming dominant. Mechanism II induced failures have a much smaller increase rate against elevating electric fields (more obviously shown later in Fig. 5), thus can be easily overshadowed by other mechanisms. The cause of mechanism II could be the extrinsic defects created during the device fabrication or a relatively high stacking fault density in the $3 \mathrm{C}-\mathrm{SiC}$ epilayer. Above $8.5 \mathrm{MV} / \mathrm{cm}$, a considerably sharp increase of the total failed devices is observed, namely mechanism III. It is worth mentioning that $8.5-9 \mathrm{MV} / \mathrm{cm}$ is also previously reported in $4 \mathrm{H}-\mathrm{SiC}$ TDDB tests as a juncture point where the failure mechanism changes [7-9]. For 4H-SiC, one of the common explanations for such change at high electric fields is F-N tunnelling. As mentioned previously, F-N tunnelling 
almost always occurred around 5-5.5 MV/cm in the case of $4 \mathrm{H}-$ $\mathrm{SiC}$, and here we have shown that it is delayed to above 7 $\mathrm{MV} / \mathrm{cm}$ for $3 \mathrm{C}-\mathrm{SiC}$. The fact they both change failure mechanisms around $8.5 \mathrm{MV} / \mathrm{cm}$ makes the $\mathrm{F}-\mathrm{N}$ tunnelling explanation not satisfactory. On the other hand, the electron impact ionization within the $\mathrm{SiO}_{2}$ is determined by the oxide thickness and electric field. For the mostly studied $\approx 50 \mathrm{~nm}$ thick oxides of power MOS devices, the impact ionization is expected to occur at an electric field strength of $\approx 8 \mathrm{MV} / \mathrm{cm}[43$, 44]. While for thin oxide layers, impact ionization occurs at higher electric fields, which also explains why even at a very high electric field of $15 \mathrm{MV} / \mathrm{cm}$, a Weibull distribution can still be observed for the TDDB results of very thin $(6-7 \mathrm{~nm}) \mathrm{SiO}_{2}$ layers on $3 \mathrm{C}-\mathrm{SiC}[16]$. As such, here we attribute mechanism III to the electron impact ionization.

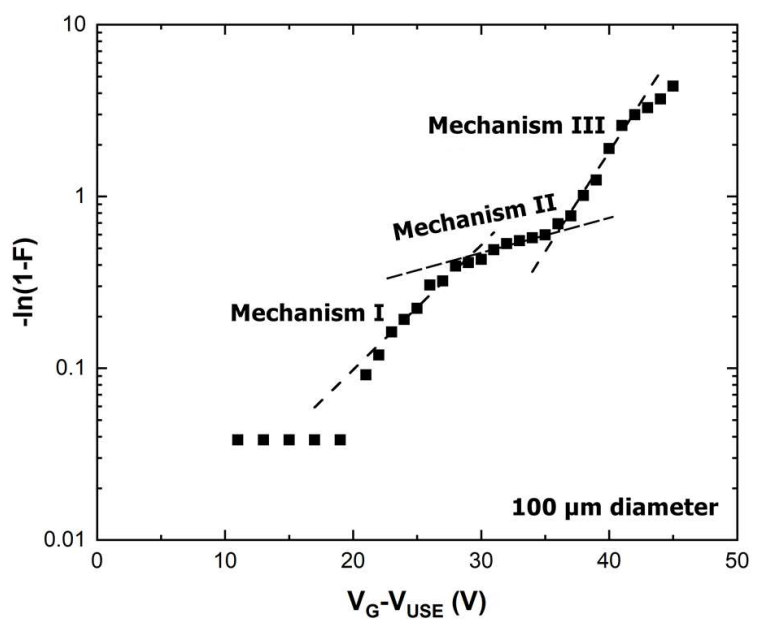

Fig. 5. Weibull distributions of $\Phi 100$ MOS capacitors as a function of applied gate bias, $\mathrm{F}$ is the failure rate and VUSE is the normal use voltage.

The three failure mechanisms can be more easily distinguished for $\Phi 100$ MOS capacitors by plotting $-\ln (1-F)$ against $V_{G^{-}} V_{U S E}$ as shown in Fig. 5.F is the failure rate and $V_{U S E}$ is the nominal voltage of the device. Since the normal operating electric field of MOS devices is typically $3 \mathrm{MV} / \mathrm{cm}[4,8]$, here we assume a $V_{U S E}$ of $20 \mathrm{~V}$. An exponential function [45] described by Eq. (2) can be used to fit the three mechanism regions as seen in Fig. 5.

$$
-\ln (1-F)=a \cdot e^{b\left(V_{G}-V_{U S E}\right)}
$$

$a$ and $b$ are the fitting parameters. By multiplying $a$ by $10^{6}$, the maximum failure parts per million (PPM) of the $\Phi 100 \mathrm{MOS}$ capacitors working for 1 hour at $3 \mathrm{MV} / \mathrm{cm}$ and room temperature can be estimated. With the low field being dominated by mechanism I, the failure rate can be estimated to be around $3450 \mathrm{PPM}$, which is close to the latest $4 \mathrm{H}-\mathrm{SiC}$ MOSFET results of 4100 PPM [45]. Although the $4 \mathrm{H}-\mathrm{SiC}$ MOSFETs were tested at a higher temperature $\left(150^{\circ} \mathrm{C}\right)$ and a longer gate bias $(168 \mathrm{~h})$, the channel length of these commercial MOSFETs usually goes down to $1 \mu \mathrm{m}$ [46], namely 10-100 times smaller gate active area than the $\Phi 100$ MOS capacitors studied here. Extrapolation from the high field $(>8.5 \mathrm{MV} / \mathrm{cm})$ data points suggests a much lower failure rate of 40 PPM, indicating a great room for further improvements if mechanism I can be minimized by improving material quality.

\section{D. $T D D B$}

Long-term TDDB test was performed on $\Phi 100$ MOS capacitors at room temperature using a custom-built dielectric breakdown rig. Gate leakage currents of up to 32 devices were monitored simultaneously in parallel, and the maximum current level was separately limited to $1 \mu \mathrm{A}$ by series connected resistors. Any device with a gate leakage increasing to above 1 $\mu \mathrm{A}$ was removed from operation to protect the adjacent devices. Literatures have suggested that the thick gate oxide breakdown is a "weakest link" type of problem [8, 47, 48], thus can be described by the Weibull distribution expression as below:

$$
F\left(T_{B D}\right)=1-e^{\left[-\left(\frac{T_{B D}}{t_{63 \%}}\right)^{\beta}\right]}
$$

where $T_{B D}$ is the TDDB running time, $t_{63} \%$ is the characterisitc time when $63 \%$ of all tested devices fail, and $\beta$ is the Weibull slope, which is an indicator of failure mechanism variance between devices and should increase with reducing number of mechanisms.

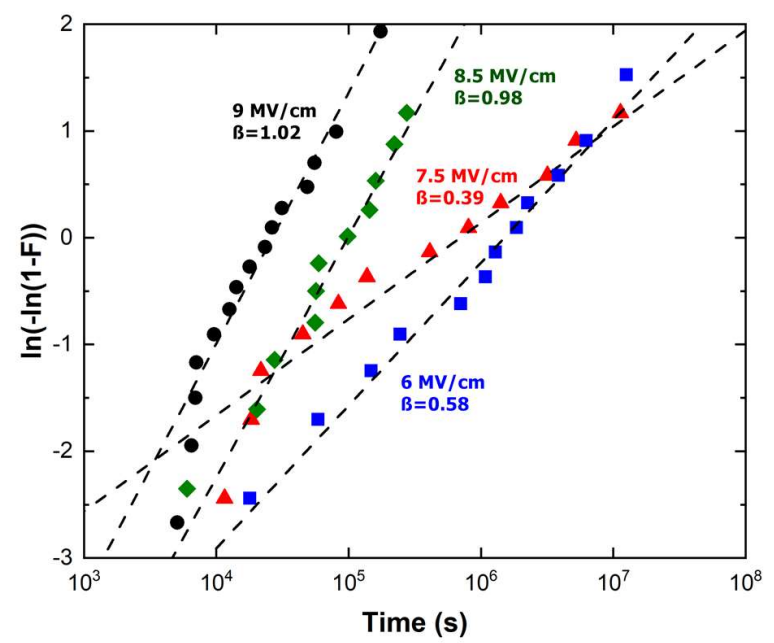

Fig. 6. Weibull distributions of devices failures at various electric fields on $\Phi 100$ MOS capacitors, obvious premature failures have been removed.

Measurements were taken for $6 \mathrm{MV} / \mathrm{cm}, 7.5 \mathrm{MV} / \mathrm{cm}, 8.5$ $\mathrm{MV} / \mathrm{cm}$ and $9 \mathrm{MV} / \mathrm{cm}$, and the Weibull distributions are shown in Fig. 6 with obvious premature failure tails removed. Even at high electric fields above $8.5 \mathrm{MV} / \mathrm{cm}$, the extracted $\beta$ values are still very low $\approx 1$, an order of magnitude smaller than the values reported for $4 \mathrm{H}-\mathrm{SiC}$ [7], suggesting that mechanism II may still be working behind the scene at such high fields. It can be seen in Fig. 6 that, the slope $\beta$ for $9 \mathrm{MV} / \mathrm{cm}$ and $8.5 \mathrm{MV} / \mathrm{cm}$ are almost the same $(\approx 1)$, whereas considerably reduced to 0.39 for $7.5 \mathrm{MV} / \mathrm{cm}$. From previous discussions, we know it is because of the change of dominating failure mechanism from II to III with elevated electric fields. Further reducing the test field to 6 $\mathrm{MV} / \mathrm{cm}$ confirmed an increase of $\beta$ to 0.58 due to mechanism I, agreeing with the medium-term distribution analysis.

To project the lifetime of the fabricated MOS capacitors, $t_{63} \%$ is plotted against the applied gate electric field (the E model 
[49]) as seen in Fig. 7. The field acceleration parameter is extracted to be $0.906 \mathrm{dec} /(\mathrm{MV} / \mathrm{cm})$ for $7.5-9 \mathrm{MV} / \mathrm{cm}$. From the projection, the lifetime of these devices can reach 10 years at room temperature with a maximum operating electric field of 4.5 MV/cm as shown in Fig. 7. Temperature has a significant impact on the life time of $\mathrm{SiO}_{2}$. Although the testing rig is limited to room temperature, higher temperature performance of these MOS capacitors can still be estimated using the Arrhenius model $t_{63 \%} \propto \exp \left(E_{a} / k T\right)$, where $E_{a}$ is the thermal activation energy. $3 \mathrm{C}$-SiC MOSFETs operating at $300{ }^{\circ} \mathrm{C}$ have been demonstrated [36], but there were no reports on the $E_{a}$ value. From the $\mathrm{Al}$ gated $4 \mathrm{H}-\mathrm{SiC}$ MOS capacitor results [50], $0.5 \mathrm{eV}$ was assumed for these $3 \mathrm{C}-\mathrm{SiC}$ devices, and the life time projection for $150{ }^{\circ} \mathrm{C}$ is shown in Fig. 7. The predicted critical electric field required for 10 year life time drops considerably to $1.4 \mathrm{MV} / \mathrm{cm}$ at $150{ }^{\circ} \mathrm{C}$, much lower than the $4 \mathrm{H}-\mathrm{SiC}$ MOS capacitors results, $\approx 6 \mathrm{MV} / \mathrm{cm}[8]$. However, it should be noted that, due to a positively charged 3C-SiC MOS interface as mentioned before, a forward gate bias accumulates more electrons at the $3 \mathrm{C}-\mathrm{SiC} / \mathrm{SiO}_{2}$ interface at room temperature compared with the $4 \mathrm{H}-\mathrm{SiC}$ case. As such, the actual temperature effects may be smaller than predicted in Fig. 7.

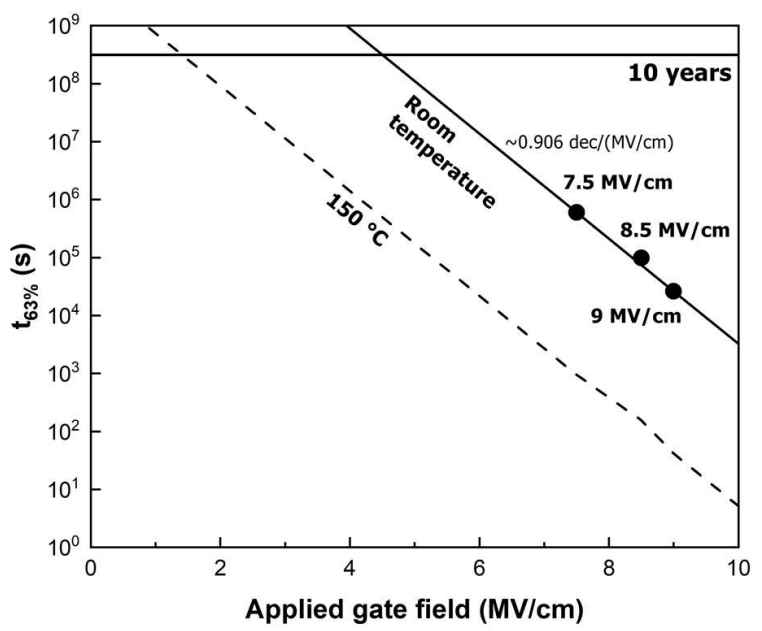

Fig. 7. Time to breakdown as a function of the applied electric field of $\Phi 100$ MOS capacitors and the projection of lifetime using the $\mathrm{E}$ model.

\section{SUMMARY}

A reliability study has been thoroughly performed on $3 \mathrm{C}-\mathrm{SiC}$ MOS capacitors at room temperature, including leakage current evaluation, medium-term constant voltage and long-term TDDB tests. The effective barrier height at $3 \mathrm{C}-\mathrm{SiC} / \mathrm{SiO}_{2}$ is experimentally obtained as very close to the theoretical value of $3.7 \mathrm{eV}$, and the F-N tunnelling is found to be dominating the gate leakage current only above a relatively high electric field of $\approx 7 \mathrm{MV} / \mathrm{cm}$. According to the medium-term reliability test at a wide electric field range, an acceptable failure rate of 3450 PPM (1 hour at $3 \mathrm{MV} / \mathrm{cm}$, room temperature) for the $100 \mu \mathrm{m}$ diameter MOS capacitors is obtained, which can be further reduced to 40 PPM if the extrinsic defects (mechanism I) are neglected. Long-term TDDB tests over several months have been done for $\Phi 100 \mathrm{MOS}$ capacitors at $7.5 \mathrm{MV} / \mathrm{cm}, 8.5 \mathrm{MV} / \mathrm{cm}$ and $9 \mathrm{MV} / \mathrm{cm}$. The field acceleration factor is found to be $\approx 0.906 \mathrm{dec} /(\mathrm{MV} / \mathrm{cm})$ in the studied range. Both the mediumterm and long-term tests show a failure mechanism change of these MOS capacitors at $8.5 \mathrm{MV} / \mathrm{cm}$, which is most likely caused by the impact ionization of electrons rather than the F$\mathrm{N}$ tunnelling. Although the fabricated MOS capacitors are still suffering from a high number of extrinsic defects and further improvements are required, the projected lifetime is still remarkable and can reach 10 years, at $4.5 \mathrm{MV} / \mathrm{cm}$ and room temperature.

\section{REFERENCES}

[1] J. Lutz, H. Schlangenotto, U. Scheuermann, and R. De Doncker, "Reliability and Reliability Testing," in Semiconductor Power Devices: Physics, Characteristics, Reliability, 2nd ed., Springer International Publishing AG, pp. 489-581, 2018. DOI: 10.1007/978-3-319-70917-8.

[2] A. K. Agarwal, S. Seshadri, and L. B. Rowland, "Temperature dependence of Fowler-Nordheim current in $6 \mathrm{H}-$ and $4 \mathrm{H}-\mathrm{SiC}$ MOS capacitors," IEEE Electron Device Lett., vol. 18, no. 12, pp. 592-594, 1997. DOI: $10.1109 / 55.644081$.

[3] L. A. Lipkin and J. W. Palmour, "Insulator investigation on SiC for improved reliability," IEEE Trans. Electron Devices, vol. 46, no. 3, pp. 525-532, 1999. DOI: 10.1109/16.748872.

[4] R. Singh and A. R. Hefner, "Reliability of SiC MOS devices," Solid-State Electron, vol. 48, no. 10, pp. 1717-1720, 2004/10/01/ 2004, DOI: $10.1016 /$ j.sse.2004.05.005.

[5] S. Krishnaswami, M. Das, B. Hull, S.H. Ryu, J. Scofield, A. Agarwal, and J. Palmour, "Gate oxide reliability of 4H-SiC MOS devices," in Proc. 43rd Annu. IEEE Int. Rel. Phys. Symp., 2005, pp. 592-593, DOI: 10.1109/RELPHY.2005.1493158.

[6] K. Matocha and R. Beaupre, "Time-Dependent Dielectric Breakdown of Thermal Oxides on 4H-SiC," Mater. Sci. Forum, vol. 556-557, pp. 675678, 2007. DOI: 10.4028/www.scientific.net/MSF.556-557.675.

[7] K. Matocha, G. Dunne, S. Soloviev, and R. Beaupre, "Time-Dependent Dielectric Breakdown of 4H-SiC MOS Capacitors and DMOSFETs," IEEE Trans. Electron Devices, vol. 55, no. 8, pp. 1830-1834, 2008, DOI: 10.1109/TED.2008.926595

[8] M. Gurfinkel, J.C. Horst, J.S. Suehle, J.B. Bernstein, Y. Shapira, K.S. Matocha, G. Dunne, and R.A. Beaupre, "Time-Dependent Dielectric Breakdown of $4 \mathrm{H}-\mathrm{SiC} / \mathrm{SiO}_{2}$ MOS Capacitors," IEEE Trans. Device and Materials Reliability, vol. 8, no. 4, pp. 635-641, 2008. DOI: 10.1109/TDMR.2008.2001182.

[9] L. C. Yu, G. T. Dunne, K. S. Matocha, K. P. Cheung, J. S. Suehle, and K. Sheng, "Reliability Issues of SiC MOSFETs: A Technology for HighTemperature Environments," IEEE Trans. Device and Materials Reliability, vol. 10 , no. 4, pp. 418-426, 2010. DOI: 10.1109/TDMR.2010.2077295.

[10] T. Watanabe, S. Hino, T. Iwamatsu, S. Tomohisa, and S. Yamakawa, "Mechanism of Depletion-Mode TDDB for 4H-SiC MOS Structure," IEEE Trans. Device and Materials Reliability, vol. 17, no. 1, pp. 163-169, 2017. DOI: 10.1109/TDMR.2016.2635160.

[11] P. Samanta and K. C. Mandal, "Leakage current conduction, hole injection, and time-dependent dielectric breakdown of n-4H-SiC MOS capacitors during positive bias temperature stress," J. Appl. Phys, vol. 121, no. 3, p. 034501, 2017. DOI: 10.1063/1.4973674.

[12] A. Xiang, X. Xu, L. Zhang, Z. Li, J. Li, and G. Dai, "Origin of temperature dependent conduction of current from n-4H-SiC into silicon dioxide films at high electric fields," Appl. Phys. Lett., vol. 112, no. 6, p. 062101, 2018. DOI: $10.1063 / 1.5006249$.

[13] J. Lutz, H. Schlangenotto, U. Scheuermann, and R. De Doncker, "MOS Transistors and Field Controlled Wide Bandgap Devices," in Semiconductor Power Devices: Physics, Characteristics, Reliability, 2nd ed., Springer International Publishing AG, pp. 341-390, 2018. DOI: https://doi.org/10.1007/978-3-319-70917-8.

[14] S. Lombardo, J. H. Stathis, B. P. Linder, K. L. Pey, F. Palumbo, and C. H. Tung, "Dielectric breakdown mechanisms in gate oxides," J. Appl. Phys, vol. 98, no. 12, p. 121301, 2005. DOI: 10.1063/1.2147714.

[15] P. Fiorenza, A. Frazzetto, A. Guarnera, M. Saggio, and F. Roccaforte, "Fowler-Nordheim tunneling at $\mathrm{SiO}_{2} / 4 \mathrm{H}-\mathrm{SiC}$ interfaces in metal-oxide- 
semiconductor field effect transistors," Appl. Phys. Lett., vol. 105, no. 14 p. 142108, 2014. DOI: 10.1063/1.4898009.

[16] J. Eriksson, F. Roccaforte, P. Fiorenza, M.H. Weng, F. Giannazzo, J. Lorenzzi, N. Jegenyes, G. Ferro and V. Raineri, "Nanoscale probing of dielectric breakdown at $\mathrm{SiO}_{2} / 3 \mathrm{C}-\mathrm{SiC}$ interfaces," J. Appl. Phys, vol. 109 no. 1, p. 013707,2011 , DOI: $10.1063 / 1.3525806$.

[17] K. Yagi, T. Kawahara, N. Hatta, and H. Nagasawa, "'Switch-Back Epitaxy'as a Novel Technique for Reducing Stacking Faults in 3C-SiC," Mater. Sci. Forum, vol. 527, pp. 291-294, 2006. DOI: 10.4028/www.scientific.net/MSF.527-529.291

[18] J. Eid, I. G. Galben, and S. Saddow, "3C-SiC growth on Si substrates via CVD: An introduction," in Physics of Advanced Materials Winter School, pp. 281-304, 2008.

[19] F. La Via, A. Severino, R. Anzalone, C. Bongiorno, G. Litrico, M. Mauceri, M. Schoeler, P. Schuh, and P. Wellmann, "From thin film to bulk 3C-SiC growth: Understanding the mechanism of defects reduction," Mat. Sci. Semicon. Proc, vol. 78, pp. 57-68, 2018. DOI 10.1016/j.mssp.2017.12.012.

[20] R. Anzalone, A. Serverino, G. D’Arrigo, C. Bongiorno, G. Abbondanza, G. Foti, S. Saddow, and F. La Via, "Heteroepitaxy of 3C-SiC on differen on-axis oriented silicon substrates," J. Appl. Phys, vol. 105, no. 8, pp. 084910-7, 2009. DOI: 10.1063/1.3095462.

[21] R. Yakimova, R. Vasiliauskas, J. Eriksson, and M. Syväjärvi, "Progress in 3C-SiC growth and novel applications," Mater. Sci. Forum, vol. 711, pp. 3-10, 2012. DOI: 10.4028/www.scientific.net/MSF.711.3.

[22] A. Gupta and C. Jacob, "Selective epitaxy and lateral overgrowth of 3C$\mathrm{SiC}$ on $\mathrm{Si}-\mathrm{A}$ review," Prog. Cryst. Growth. Ch, vol. 51, no. 1-3, pp. $43-$ 69, 2005. DOI: 10.1016/j.pcrysgrow.2005.10.003.

[23] N. Hatta, T. Kawahara, K. Yagi, H. Nagasawa, S. A. Reshanov, and A Schöner, "Reliable method for eliminating stacking fault on $3 \mathrm{C}-\mathrm{SiC}$ (001)," Mater. Sci. Forum, vol. 717, pp. 173-176, 2012

[24] H. Nagasawa and K. Yagi, "3C-SiC single-crystal films grown on 6-Inch Si substrates," Physica Status Solidi (b), vol. 202, no. 1, pp. 335-358, 1997. DOI: 10.1002/1521-3951(199707)202:1<335::AIDPSSB335>3.0.CO;2-Y.

[25] X. Song, J. Biscarrat, A.E. Bazin, J.F. Michaud, F. Cayrel, M. Zielinski, T. Chassagne, M. Portail, E. Collard, and D. Alquier, "Dose Influence on Physical and Electrical Properties of Nitrogen Implantation in 3C-SiC on Si," Mater. Sci. Forum, vol. 711, pp. 154-158, 2012. DOI: 10.4028/www.scientific.net/MSF.711.154

[26] F. Li, Y. Sharma, V. Shah, M.R. Jennings, A. Perez-Tomas, M. Myronov, C.A. Fisher, D. Leadley, and P.A. Mawby, "Electrical activation of nitrogen heavily implanted 3C-SiC(1 00$)$," Applied Surface Science, vol. 353, pp. 958-963, 2015. DOI: 10.1016/j.apsusc.2015.06.169.

[27] Y. K. Sharma, F. Li, M.R. Jennings, C.A. Fisher, A. Perez-Tomas, S Thomas, D.P. Hamilton, S.A.O. Russell, and P.A. Mawby "Hightemperature $\left(1200-1400^{\circ} \mathrm{C}\right)$ dry oxidation of $3 \mathrm{C}-\mathrm{SiC}$ on silicon," Journa of Electronic Materials, vol. 44, no. 11, pp. 4167-4174, 2015. DOI 10.1007/s11664-015-3949-4

[28] R. Esteve, A. Schoner, S. A. Reshanov, C. M. Zetterling, and H. Nagasawa, "Comparative study of thermally grown oxides on n-type free standing 3C-SiC (001)," J. Appl. Phys, vol. 106, no. 4, pp. 044513-4, 2009. DOI: $10.1063 / 1.3204642$.

[29] B. Zippelius, M. Hauck, S. Beljakowa, H.B. Weber, M. Krieger, H. Nagasawa, H. Uchida, G. Pensl, and A. Schoner, "Dependence of the channel mobility in 3C-SiC n-MOSFETs on the crystal orientation and channel length," Mater. Sci. Forum, vol. 717, pp. 1113-1116, 2012. DOI: 10.4028/www.scientific.net/MSF.717-720.1113.

[30] J. Eriksson, M. H. Weng, F. Roccaforte, F. Giannazzo, S. Leone, and V. Raineri, "Toward an ideal Schottky barrier on 3C-SiC," Appl. Phys. Lett., vol. 95, no. 8, pp. 081907-3, 2009. DOI: 10.1063/1.3211965

[31] F. Li, Y. Sharma, D. Walker, S. Hindmarsh, M.R. Jennings, D. Martin, C.A. Fisher, P.M. Gammon, A. Perez-Tomas, and P.A. Mawby, "3C-SiC transistor with ohmic contacts defined at room temperature," IEEE Electron Device Letters, vol. 37, no. 9, pp. 1189-1192, 2016. DOI: 10.1109/LED.2016.2593771.

[32] H. Nagasawa, M. Abe, K. Yagi, T. Kawahara, and N. Hatta, "Fabrication of high performance $3 \mathrm{C}-\mathrm{SiC}$ vertical MOSFETs by reducing planar defects," Physica Status Solidi (b), vol. 245, no. 7, pp. 1272-1280, 2008 DOI: $10.1002 / \mathrm{pssb} .200844053$.

[33] T. Gutt, T. Malachowski, H. M. Przewlocki, O. Engstrom, M. Bakowski, and R. Esteve, "Investigation of gate edge effect on interface trap density in 3C-SiC MOS capacitors," Materials Science and Engineering BAdvanced Functional Solid-State Materials, vol. 177, no. 15, pp. $1327-$ 1330, 2012. DOI: 10.1016/j.mseb.2012.03.007
[34] M. Krieger, S. Beljakowa, L. Trapaidze, T. Frank, H.B. Weber, G. Pensl, N. Hatta, M. Abe, H. Nagasawa, and A. Schoner, "Analysis of interface trap parameters from double-peak conductance spectra taken on nimplanted 3C-SiC MOS capacitors," Physica Status Solidi (b), vol. 245, no. 7, pp. 1390-1395, 2008. DOI: 10.1002/pssb.200844062.

[35] F. Li, Y.K.Sharma, M.R. Jennings, A. Perez-Tomas, V.A. Shah, H. Rong, S.A.O. Russell, D.M. Martin, and P.A. Mawby, "Improved channel mobility by oxide nitridation for n-channel MOSFET on 3C-SiC (100)/Si," Mater. Sci. Forum, vol. 858, pp. 667-670, 2016. DOI: 10.4028/www.scientific.net/MSF.858.667.

[36] H. Uchida, A. Minami, T. Sakata, H. Nagasawa, and M. Kobayashi, "High temperature performance of 3C-SiC MOSFETs with high channel mobility," Mater. Sci. Forum, vol. 717, pp. 1109-1112, 2012. DOI: 10.4028/www.scientific.net/MSF.717-720.1109

[37] K.K. Lee, Y. Ishida, T. Ohshima, K. Kojima, Y. Tanaka, T. Takahashi, H. Okumura, K. Arai, and T. Kamiya, "N-channel MOSFETs fabricated on homoepitaxy-grown 3C-SiC films," IEEE Electron Device Letters, vol. 24, no. 7, pp. 466-468, 2003. DOI: 10.1109/LED.2003.815006.

[38] V. V. Afanas' ev, F. Ciobanu, S. Dimitrijev, G. Pensl, and A. Stesmans, "SiC/SiO interface states: properties and models," Mater. Sci. Forum, vol. 483, pp. 563-568, 2005. DOI: 10.4028/www.scientific.net/MSF.483485.563.

[39] A. V. Penumatcha, S. Swandono, and J. A. Cooper, "Limitations of the high-low $\mathrm{C}-\mathrm{V}$ technique for MOS interfaces with large time constant dispersion," IEEE Trans. Electron Devices, vol. 60, no. 3, pp. 923-926, 2013. DOI: 10.1109/TED.2013.2237777.

[40] R. Arora, J. Rozen, D.M. Fleetwood, K.C. Galloway, C.X. Zhang, J. Han, S. Dimitrijev, F. Kong, L.C. Feldman, S.T. Pantelides, and R.D. Schrimpf, "Charge trapping properties of $3 \mathrm{C}$ - and $4 \mathrm{H}-\mathrm{SiC}$ MOS capacitors with nitrided gate oxides," IEEE Trans. Nuclear Science, vol. 56, no. 6, pp. 3185-3191, 2009. DOI: 10.1109/TNS.2009.2031604.

[41] M. Lenzlinger and E. H. Snow, "Fowler-Nordheim tunneling into thermally grown $\mathrm{SiO}_{2}$," J. Appl. Phys, vol. 40, no. 1, pp. 278-283, 1969. DOI: $10.1063 / 1.1657043$.

[42] J. Senzaki, K. Kojima, T. Kato, A. Shimozato, and K. Fukuda, "Correlation between reliability of thermal oxides and dislocations in ntype 4H-SiC epitaxial wafers," Appl. Phys. Lett., vol. 89, no. 2, p. 022909 , 2006, DOI: $10.1063 / 1.2221525$.

[43] U. Schwalke, M. Pölzl, T. Sekinger, and M. Kerber, "Ultra-thick gate oxides: charge generation and its impact on reliability," Microelectronics Reliability, vol. 41, no. 7, pp. 1007-1010, 2001. DOI: 10.1016/S00262714(01)00058-0.

[44] D. Arnold, E. Cartier, and D. J. DiMaria, "Theory of high-field electron transport and impact ionization in silicon dioxide," Physical Review B, vol. 49, no. 15, pp. 10278-10297, 1994. DOI: 10.1103/PhysRevB.49.10278.

[45] M. Beier-Moebius and J. Lutz, "Breakdown of gate oxide of $1.2 \mathrm{kV} \mathrm{SiC}$ MOSFETs under high temperature and high gate voltage," in PCIM Europe 2016, pp. 1-8.

[46] P. A. Losee, K. Matocha, S.D. Arthur, J. Nasadoski, Z. Stum, J.L. Garrett, M. Schutten, G. Dunne, and L. Stevanovic, "DC and transient performance of $4 \mathrm{H}-\mathrm{SiC}$ double-implant MOSFETs," IEEE Trans. Electron Devices, vol. 55, no. 8, pp. 1824-1829, 2008. DOI: 10.1109/TED.2008.926592.

[47] J. Sune, "New physics-based analytic approach to the thin-oxide breakdown statistics," IEEE Electron Device Letters, vol. 22, no. 6, pp. 296-298, 2001. DOI: 10.1109/55.924847.

[48] J. H. Stathis, "Percolation models for gate oxide breakdown," J. Appl. Phys, vol. 86, no. 10, pp. 5757-5766, 1999. DOI: 10.1063/1.371590.

[49] J. W. McPherson and D. A. Baglee, "Acceleration factors for thin gate oxide stressing," in in Proc. 23rd Annu. IEEE Int. Rel. Phys. Symp., pp. 1-5, 1985. DOI: 10.1109/IRPS.1985.362066

[50] J. Senzaki, A. Shimozato, and K. Fukuda, "Acceleration factors in acceleration life test of thermal oxides on $4 \mathrm{H}-\mathrm{SiC}$ wafers," Mater. Sci. Forum, vol. 556-557, pp. 635-638, 2007. DOI: 10.4028/www.scientific.net/MSF.556-557.635. 